\title{
Multifocal and Multicentric Breast Carcinoma: A Significantly More Aggressive Tumor than Unifocal Breast Cancer
}

\author{
ZHIQIANG LANG ${ }^{1 *}$, YANQIU WU ${ }^{2 *}$, CUIYAN LI $^{2}$, XINNA LI $^{1}$, XUAN WANG $^{2}$ and GUIMEI QU ${ }^{1}$ \\ Departments of ${ }^{1}$ Pathology and ${ }^{2}$ Medicine, Yuhuangding Hospital of Qingdao University, Yantai, P.R. China
}

\begin{abstract}
Background/Aim: There are still many questions that surround multifocal or multicentric breast carcinoma $(M M B C)$. The aim of this study was to analyze the clinicopathological characteristics of MMBC and provide feasible suggestions for therapy. Patients and Methods: A total of 156 cases of MMBC in 3,597 invasive ductal breast carcinomas were collected and reviewed. Some factors related with prognosis such as tumor size, lymph node metastasis and others were assessed in each tumor focus, and mismatches among foci were recorded. Results: The majority of $M M B C$ had aggregate dimensions over $2 \mathrm{~cm}$ (85.90\%). The rate of axillary lymph node metastasis was $56.41 \%$ (88/156) compared to unifocal tumors of $33.01 \%(1,136 / 3,441)$. Most cases had higher Ki-67 proliferative indices (91/156). Mismatches in ER status were present in 6 cases, $P R$ in 4 cases, proliferative index (Ki-67) in 9 cases and HER2positive status in 2 cases. Conclusion: The larger aggregate dimension of tumor, the higher metastatic rate of axillary lymph node and the high Ki-67 proliferative index seen in most cases, suggest that MMBC is biologically more aggressive than unifocal breast cancer. In addition, every focus should be tested owing to the existence of different expressions of immunostaining between foci.
\end{abstract}

Breast carcinoma is the most prevalent malignant disease in the world. With the gradually increasing incidence and the development of mammography, more and more foci are found to be ipsilateral, which were defined as multifocal and multicentric breast carcinoma (MMBC) (1-4). In developed

\footnotetext{
*These Authors contributed equally to this study.

Correspondence to: Xuan Wang and Guimei Qu, Departments of pathology and medicine, Yuhuangding Hospital of Qingdao University, Yantai, Shandong Province, P.R. China. Tel: +86 13589883152,e-mail: anttylo@163.com,dls3152@163.com

Key Words: Multifocal and multicentric breast carcinoma, ER, PR, HER2.
}

countries the incidence of MMBC has been reported to be between $9-75 \%$ depending on the diagnostic criteria applied (5), while in China it is only around $2 \%$ according to a recent multicenter study (6). One of the most important reasons for this difference is the lack of uniform standardization. Some researchers defined MMBC as cancer with multiple invasive foci (of any size) separated by at least $4 \mathrm{~mm}$ of normal breast tissue, while more studies use the definition of "more than one focus of invasive carcinoma separated by benign tissue whether in the same or a different quadrant and regardless of the distance between the foci" (7). In addition, there are various clinical standards and mammography methods. Although great amount of work remains to be done to study the clinico-pathological features and related factors of prognosis, no agreement has been achieved. The aim of this study was to analyze the clinicopathological features, observe the differences between different foci and provide feasible suggestions for clinical treatment.

\section{Patients and Methods}

Patients. Data from patients with the diagnosis of invasive ductal carcinoma from Yantai Yuhuangding Hospital between January 2005 and June 2016 were reviewed and 156 cases of MMBC were confirmed by two experienced pathologists. MMBC was defined as two or more invasive carcinomas separated by benign tissue whether in the same or a different quadrant and regardless of the distance between the foci. Only the cases with invasive ductal carcinoma were included and multiple lesions with different histological features in different breasts were excluded. Age, location, number of foci, size of the tumor and grade, lymph node metastasis status and expression of estrogen receptor (ER), progesterone receptor (PR), HER2, Ki67 proliferative index and Ecadherin were recorded. All breast cancer specimens were routinely analyzed by pathologists according to the World Health Organisation (WHO) (2012) guidelines.

Methods. Immunohistochemical methods were used to assess the status of ER, PR, HER2, Ki67 and E-cadherin. The ER and PR staining was further classified as 'positive' (staining of at least $1 \%$ of cells) or 'negative'. HER2 positive staining was defined according to the American Society of Clinical Oncology (ASCO)/College of American Pathologists (CAP) guidelines (2013) (8). Ki67 staining was labeled 'high' (in at least $20 \%$ of the cells) 
or 'low'. E-cadherin staining was classified as 'positive' (staining of at least $10 \%$ of cells) or 'negative'. In addition, all differences between different foci were recorded and compared. Moreover, the HER2 gene was detected in each mass by fluorescence in situ hybridization (FISH) with HER2 immunohistochemical mismatches according to the new guidelines from the ASCO/CAP(8). All of the experiments were processed using licensed and validated reagents and methods in the same laboratory. The antibody used for HER2 immunohistochemistry was provide by Roche Group (4B5) and the experiments of FISH were accomplished using PathVysion ${ }^{\text {TM }}$ HER2 DNA Probe Kit.

\section{Results}

From January 2005 to June 2016, 3,697 cases of invasive ductal breast carcinoma received surgical treatment, of which 156 cases $(4.34 \%)$ were MMBC. Patients were aged from 3680 years (average age 50.94 years, mean $=53$ years). One hundred and thirty six of 156 cases $(87.18 \%)$ had two foci and most cases had aggregate dimensions over $2 \mathrm{~cm}(85.90 \%$, 134/156). The number and location of foci in the right and left breasts was almost equal. Invasive ductal carcinoma was present in all cases and the number of WHO grade I, II and III were 24, 78 and 54 respectively. Most cases were assessed at a higher rate of the Ki-67 proliferation index (91/156, $58.33 \%)$ and more than half of the cases $(56.41 \%, 88 / 156)$ had axillary lymph node metastasis. In particular, 2 cases were metastatic in all lymph nodes, with 16 and 22 lymph nodes respectively (Table I). Mismatches in ER status were present in 6 cases, PR in 4 cases, proliferative index (Ki-67) in 9 cases and HER 2 status in 2 cases. All cases were strongly positive for expression of E-cadherin. Mismatches in tumor grading were present in 6 cases. The majority of cases showed positive expression of ER $(112 / 156,71.80 \%)$ and PR $(101 / 156,64.74 \%)$ (Table II and Figure 1).

In addition, one case with discordant HER2 staining (larger focus $1+/$ smaller focus $3+$ ) received testing for the HER2 gene by FISH. The result demonstrated that the HER2 gene amplification (HER2/CEP17=4.33) existed in the smaller focus $(1.2 * 1.1 \mathrm{~cm})$ with negative amplification (HER2/CEP17=1.41) in the larger one $(4 * 1.5 \mathrm{~cm})$ (Figure 2$)$.

\section{Discussion}

The presence of multiple foci of unilateral breast has long been recognized (9-11), and its significance debated (3). The reported incidence of MMBC varies widely in the literature, owing to the different methods used to detect it and the definition applied. Controversial features include the definition, the incidence, the biological characteristics, prognostic significance and the ensuing implications for therapy, particularly surgical therapy (12-16). MMBC has a reported incidence of $9-75 \%$ (5). This wide range of reported incidences also reflects the lack of a standard definition of
Table I. Patient clinico-pathological characteristics.

\begin{tabular}{lc}
\hline & $n(\%)$ \\
\hline $\begin{array}{l}\text { Age (mean=53; range=36-80), years } \\
\quad<50\end{array}$ & $72(46.15)$ \\
$\geq 50$ & $84(53.85)$ \\
Number of foci & \\
2 & $136(87.18)$ \\
3 & $20(12.82)$ \\
Location & \\
Left mammary & $79(50.64)$ \\
$\quad$ Right mammary & $77(49.36)$ \\
Grade & \\
I & $24(15.38)$ \\
II & $78(50.00)$ \\
III & $54(34.62)$ \\
Axillary lymph node metastasis & \\
Positive & $88(56.41)$ \\
Negative & $68(43.59)$ \\
Ki-67 proliferation index & \\
$\quad 20 \%$ & $91(58.33)$ \\
$<20 \%$ & $65(41.67)$ \\
\hline
\end{tabular}

Table II. Mismatches in biological features among foci of MMBC.

\begin{tabular}{lcc}
\hline & Number & Rate $(\%)$ \\
\hline Mismatches in ER & 6 & 3.85 \\
Mismatches in PR & 4 & 2.56 \\
Mismatches in grading & 6 & 3.85 \\
Mismatches in Ki-67 & 9 & 5.77 \\
Mismatches in HER2 & 2 & 1.28 \\
\hline
\end{tabular}

MMBC. Many types of tumors could be seen in MMBC, including invasive ductal carcinoma, lobular neoplasia, ductal carcinoma in situ and others. Our study may present a few differences in some aspects compared to similar research in the same field due to the above reasons. Here, we focused on the features of invasive ductal carcinoma in MMBC due to the rarity of other types.

Tumor size is a well-recognized prognostic factor in breast cancer, but multifocality is not included as a prognostic factor in the earlier American Joint Committee on Cancer /Union for International Cancer Control (AJCC/UICC) system. The largest study to assess multifocality as a prognostic factor was reported by Joergensen et al. (2008) (17). A number of studies have consistently shown that multifocality is associated with a higher risk of nodal involvement than unifocal disease $(12,18,19)$. The current staging system implies that each tumor which arises independently should be used to estimate the patient's prognosis, based on the size of the largest deposit $(12,18$ - 


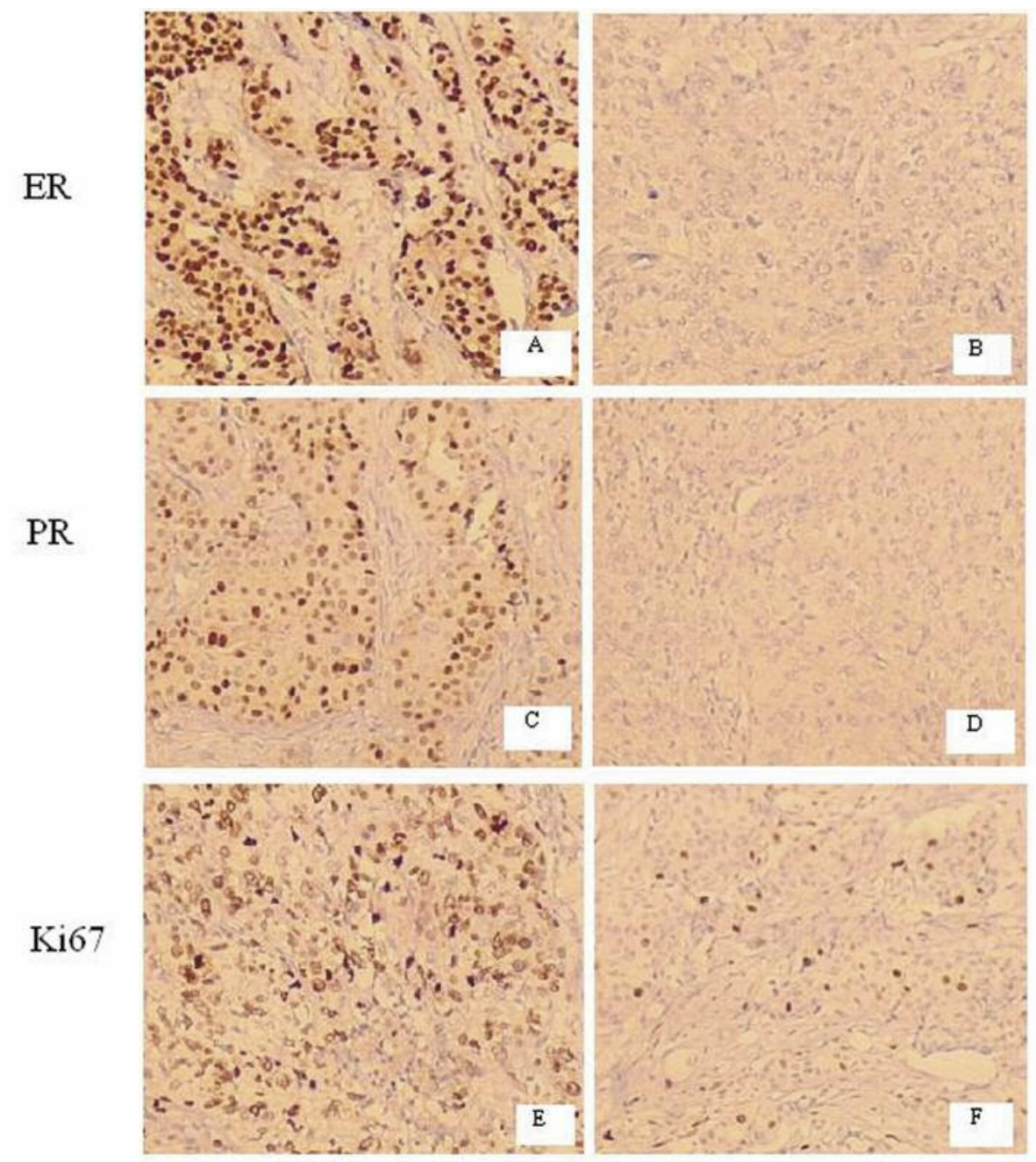

Figure 1. Immunohistochemical demonstration of ER, $P R$ and Ki67 expression in MMBC. The expression is different in different foci. ER, $P R$ and Ki67 were all expressed in the cell nucleus. The expression of $E R(A)$ is different from $(B)$; the expression of PR $(C)$ is different from $(D)$; the Ki67 proliferation index $(E)$ is different from $(F)$.

21). The current WHO guidelines (2012) agree with this opinion. This may be incorrect because of a higher risk of axillary nodal metastasis associated with multiple foci, which is in turn associated with a higher risk of local relapse (22$24)$, and a poorer prognosis $(9,21,25)$. Our data showed that the size of most foci was smaller than $2 \mathrm{~cm}$ (the clinical stage was $58.97 \%, 38.46 \%$ and $2.56 \%$ respectively), which seems be inconsistent with the high metastatic lymph node rate. While the fact that more than half of the cases $(85.90 \%$, 134/156) had aggregate dimensions over $2 \mathrm{~cm}$ (the clinical stage was $14.10 \%, 72.44 \%, 13.46 \%$ respectively) could support the aggressive behaviour of MMBC. Thus, we suggest that all foci of MMBC should be considered to estimate the risk of recurrence and metastasis. Our findings are in line with the results of Boyages et al. (26).
In addition, compared to unifocal tumors the higher rate of axillary nodal metastasis (56.41\%:33.01\%) and Ki67 proliferation index (58.33\%:42.96\%) (Table III) also indicated a poorer outcome of MMBC, although not enough follow-up data were obtained. Many earlier studies have suggested that MMBC has a negative impact on prognosis $(27,28)$, while the latest study of Neri et al., (2015) demonstrated that MMBC was an independent prognostic factor for breast cancer-specific survival together with higher numbers of metastatic axillary nodes, absence of estrogen receptors and high proliferative activity (29). Therefore, no matter how much the impact on prognosis was, MMBC should behave more aggressively.

Hormone receptor and HER2 status is used to identify patients suitable for adjuvant therapy and has prognostic 


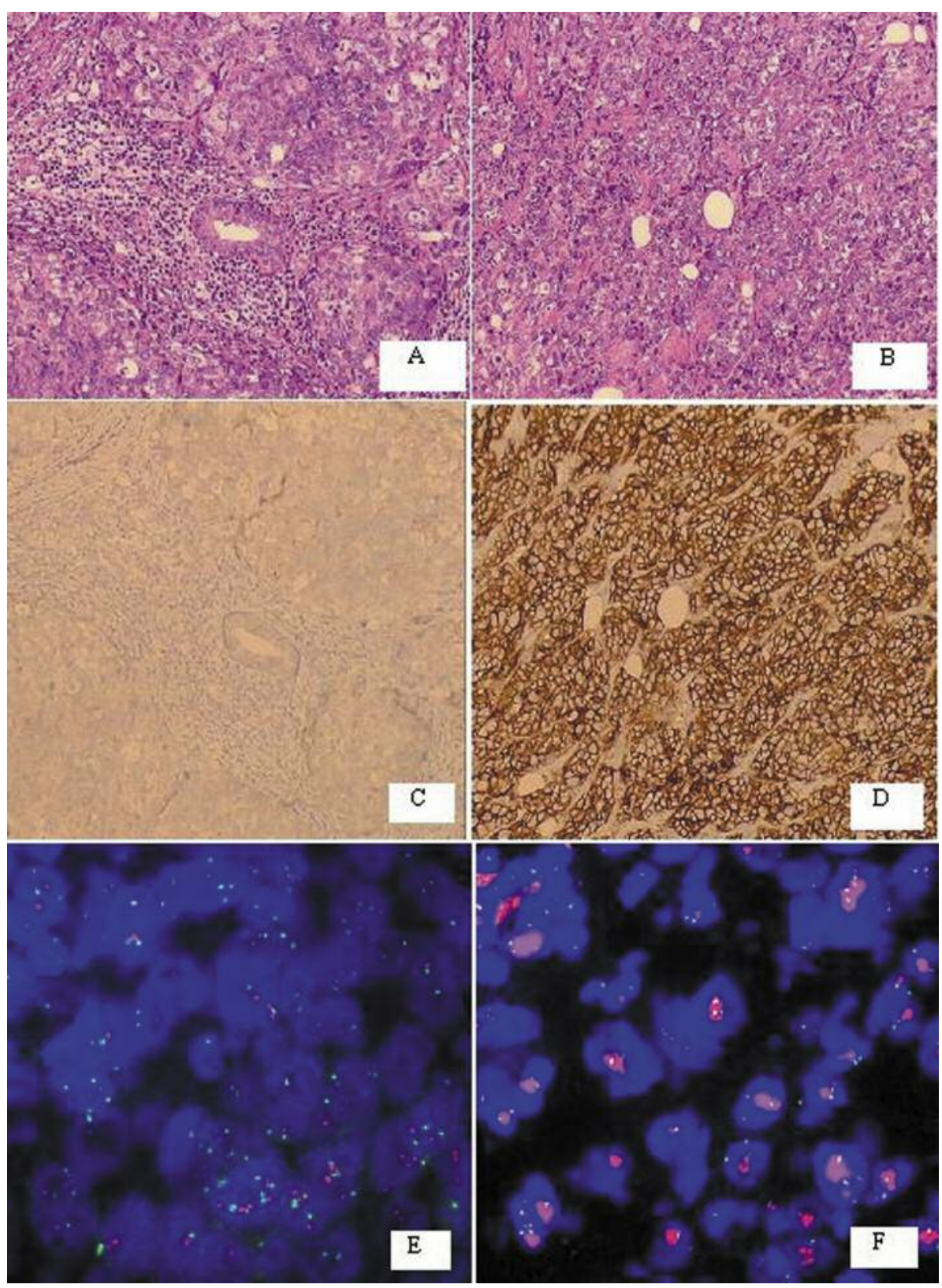

Figure 2. Both the two foci in one case were grade III (A: the larger focus; B: the smaller focus). The lager focus showed HER2 (1+) (C) and the smaller one $(3+)(D)$ by immunohistochemistry. HER2 testing showed negativity of the larger focus $(E)$ and positivity of the smaller one $(F)$ by FISH.

implications (30). However, the variability of ER, PR and HER2 status between individual foci in MMBC has not been widely studied. According to current guidelines, ER/PR status should be assessed on the sample obtained at initial core biopsy or the main tumor foci in multifocal breast cancer (more than one distinct tumor foci in a quadrant). In our study we assessed receptor status of all individual foci in $\mathrm{MMBC}$, and confirmed the variability
Table III. Comparison between MMBC and unifocal IDC.

\begin{tabular}{lcc}
\hline & MMBC (\%) & Unifocal IDC (\%) \\
\hline Axillary lymph node metastasis & 56.41 & 33.01 \\
Ki67 proliferation index over 20\% & 58.33 & 42.96 \\
HER2(3+) & 36.54 & 18.02 \\
ER(-) & 28.20 & 19.99 \\
Tumor size over 2 cm & 85.90 & 46.99 \\
\hline
\end{tabular}


similarly to another study (31). To better identify how many blocks are enough for HER2 testing in cases of MMBC, Bethune et al., (2013), examined 246 consecutive cases by immunohistochemistry and FISH (32). Their results suggest evaluating HER2 on the largest focus is enough unless the smaller focus was either a higher grade or histologically different. However, we found an exception in our cases. There were two lesions in the upper outer quadrant of the left breast of a 52-year-old female. Both lesions were invasive ductal carcinoma grade III according to WHO guidelines (2012) and ER/PR were all negative. However, HER 2 was $1+$ on the lager focus $(4 \times 1.5 \mathrm{~cm})$ and $3+$ on the smaller one $(1.2 \times 1.1 \mathrm{~cm})$. Further study by FISH showed the compatible results with immunohistochemistry. The HER2/CEP17 ratio was 1.41 on the larger and 4.33 on the smaller focus. It is clear that the larger should be considered as negative, while the smaller was positive according to the current ASCO/CAP guidelines (2013) (6). Therefore, some positive cases may be missed if detecting the largest focus only. We suggest that all individual foci should be tested in MMBC cases for better therapy and prognosis. The reasons most possible to explain the mismatch between foci were heterogeneity (31) or different clonality among tumor foci (33). However, testing every focus of MMBC to assess the gene abnormality and the difference of clonality does not seem to be an efficient approach, so before a new guideline concerning MMBC is published, immunohistochemistry is still the best way to identify HER2 status for further treatment, considering the experimental and economic level, especially in developing countries.

E-cadherin is an important cell adhesion molecule. Much research has demonstrated that down-regulation of Ecadherin is associated with breast cancer progression, poorer outcome and resistance to therapy (34, 35). Weissenbacher et al., (2013), stated that E-cadherin expression was significantly lower in MMBC compared to the unifocal group and suggested the down-regulation of Ecadherin in MMBC was causally connected with a worse prognosis (36). While our results showed that E-cadherin showed strong positive staining in all foci of $\mathrm{MMBC}$, no down-regulation was found. Therefore, more research is necessary to elucidate the molecular mechanisms of MMBC.

Although we failed to collect the follow-up data and the tumor size was usually smaller than $2 \mathrm{~cm}$, the larger aggregate dimension, higher rate of lymph node metastasis and Ki67 proliferative index and higher ER-negative rate than unifocal breast cancer suggests a more aggressive behaviour of MMBC. The variability of ER, PR and HER2 status support the idea that all individual foci should be tested in MMBC cases in order to provide the best therapy and prognosis.

\section{Conflicts of Interest}

The Authors have no conflicting or competing interests.

\section{Acknowledgements}

The Authors would like to thank Lei Jiang and Li Zhang from Yantai Yuhuangding Hospital, Yantai, P.R.China, for their help in the experiments of immunohistochemistry and FISH.

\section{References}

1 Gupta D, Nath M and Layfield LJ: Utility of four-quadrant random sections in mastectomy specimens. Breast J 9: 307-311, 2003.

2 Kumar R, Potenta S and Alavi A: Sentinel lymph node biopsy in multifocal and multicentric breast cancer. J Am Coll Surg 198: 674-676, 2004

3 Rakovitch E, Pignol JP, Hanna W, Narod S, Spayne J, NofechMozes S, Chartier C and Paszat L: Significance of multifocality in ductal carcinoma in situ: outcomes of women treated with breast-conserving therapy. J Clin Oncol 25: 5591-5596, 2007.

4 Sikand K, Lee AH, Pinder SE, Elston CW and Ellis IO: Sections of the nipple and quadrants in mastectomy specimens for carcinoma are of limited value. J Clin Pathol 58: 543-545, 2005.

5 Jain S, Rezo A, Shadbolt B and Dahlstrom JE: Synchronous multiple ipsilateral breast cancers: implications for patient management. Pathology 41: 57-67, 2009.

6 Zhou MR, Tang ZH, Li J, Fan JH, Pang Y, Yang HJ, Zheng S, Bai JQ, Lv N, Qiao YL, Qi HZ and Xu F: Clinical and pathologic features of multifocal and multicentric breast cancer in chinese women: a retrospective cohort study. J Breast Cancer 16: 77-83, 2013.

7 Cserni G, Bori R, Sejben I, Voros A, Kaiser L, Hamar S, Csorgo $\mathrm{E}$ and Kulka J: Unifocal, multifocal and diffuse carcinomas: a reproducibility study of breast cancer distribution. Breast 22: 3438, 2013.

8 Wolff AC, Hammond ME, Hicks DG, Dowsett M, McShane LM, Allison KH, Allred DC, Bartlett JM, Bilous M, Fitzgibbons P, Hanna W, Jenkins RB, Mangu PB, Paik S, Perez EA, Press MF, Spears PA, Vance GH, Viale G, Hayes DF, American Society of Clinical $\mathrm{O}$ and College of American $\mathrm{P}$ : Recommendations for human epidermal growth factor receptor 2 testing in breast cancer: American Society of Clinical Oncology/College of American Pathologists clinical practice guideline update. J Clin Oncol 31: 3997-4013, 2013.

9 Egan RL: Multicentric breast carcinomas: clinical-radiographicpathologic whole organ studies and 10-year survival. Cancer 49: 1123-1130, 1982.

10 Gallager HS and Martin JE: Early phases in the development of breast cancer. Cancer 24: 1170-1178, 1969.

11 Holland R, Veling SH, Mravunac $\mathrm{M}$ and Hendriks JH: Histologic multifocality of Tis, T1-2 breast carcinomas. Implications for clinical trials of breast-conserving surgery. Cancer 56: 979-990, 1985.

12 Coombs NJ and Boyages J: Multifocal and multicentric breast cancer: does each focus matter? J Clin Oncol 23: 7497-7502, 2005.

13 Fisher B: Role of science in the treatment of breast cancer when tumor multicentricity is present. J Natl Cancer Inst 103: 12921298, 2011. 
14 Fisher ER, Sass R, Fisher B, Wickerham L and Paik SM: Pathologic findings from the National Surgical Adjuvant Breast Project (protocol 6). I. Intraductal carcinoma (DCIS). Cancer 57: 197-208, 1986

15 Tot T: Clinical relevance of the distribution of the lesions in 500 consecutive breast cancer cases documented in large-format histologic sections. Cancer 110: 2551-2560, 2007.

16 Vaidya JS, Vyas JJ, Chinoy RF, Merchant N, Sharma OP and Mittra I: Multicentricity of breast cancer: whole-organ analysis and clinical implications. Br J Cancer 74: 820-824, 1996.

17 Joergensen LE, Gunnarsdottir KA, Lanng C, Moeller S and Rasmussen BB: Multifocality as a prognostic factor in breast cancer patients registered in Danish Breast Cancer Cooperative Group (DBCG) 1996-2001. Breast 17: 587-591, 2008.

18 Chua B, Ung O, Taylor R and Boyages J: Frequency and predictors of axillary lymph node metastases in invasive breast cancer. ANZ J Surg 71: 723-728, 2001.

19 Fowble B, Yeh IT, Schultz DJ, Solin LJ, Rosato EF, Jardines L, Hoffman J, Eisenberg B, Weiss MC and Hanks G: The role of mastectomy in patients with stage I-II breast cancer presenting with gross multifocal or multicentric disease or diffuse microcalcifications. Int J Radiat Oncol Biol Phys 27: 567-573, 1993.

20 Andea AA, Wallis T, Newman LA, Bouwman D, Dey J and Visscher DW: Pathologic analysis of tumor size and lymph node status in multifocal/multicentric breast carcinoma. Cancer 94: 1383-1390, 2002.

21 Rezo A, Dahlstrom J, Shadbolt B, Rodins K, Zhang Y, Davis AJ, Act and Sensw B: Tumor size and survival in multicentric and multifocal breast cancer. Breast 20: 259-263, 2011.

22 Katz A, Strom EA, Buchholz TA, Theriault R, Singletary SE and McNeese MD: The influence of pathologic tumor characteristics on locoregional recurrence rates following mastectomy. Int $\mathrm{J}$ Radiat Oncol Biol Phys 50: 735-742, 2001.

23 Kurtz JM, Jacquemier J, Amalric R, Brandone H, Ayme Y, Hans $\mathrm{D}$, Bressac C and Spitalier JM: Breast-conserving therapy for macroscopically multiple cancers. Ann Surg 212: 38-44, 1990.

24 Leopold KA, Recht A, Schnitt SJ, Connolly JL, Rose MA, Silver $B$ and Harris JR: Results of conservative surgery and radiation therapy for multiple synchronous cancers of one breast. Int $\mathrm{J}$ Radiat Oncol Biol Phys 16: 11-16, 1989.

25 Rakowsky E, Klein B, Kahan E, Derazne E and Lurie H: Prognostic factors in node-positive operable breast cancer patients receiving adjuvant chemotherapy. Breast Cancer Res Treat 21: 121-131, 1992.

26 Boyages J, Jayasinghe UW and Coombs N: Multifocal breast cancer and survival: each focus does matter particularly for larger tumours. Eur J Cancer 46: 1990-1996, 2010.
27 Litton JK, Eralp Y, Gonzalez-Angulo AM, Broglio K, Uyei A, Hortobagyi GN and Arun B: Multifocal breast cancer in women $<$ or =35 years old. Cancer 110: 1445-1450, 2007.

28 Vlastos G, Rubio IT, Mirza NQ, Newman LA, Aurora R, Alderfer J, Buzdar AU and Singletary SE: Impact of multicentricity on clinical outcome in patients with T1-2, N0-1, M0 breast cancer. Ann Surg Oncol 7: 581-587, 2000.

29 Neri A, Marrelli D, Megha T, Bettarini F, Tacchini D, De Franco L and Roviello F: "Clinical significance of multifocal and multicentric breast cancers and choice of surgical treatment: a retrospective study on a series of 1158 cases". BMC Surg 15: 1, 2015.

30 Regan MM and Gelber RD: Predicting response to systemic treatments: learning from the past to plan for the future. Breast 14: 582-593, 2005.

31 Buggi F, Folli S, Curcio A, Casadei-Giunchi D, Rocca A, Pietri E, Medri L and Serra L: Multicentric/multifocal breast cancer with a single histotype: is the biological characterization of all individual foci justified? Ann Oncol 23: 2042-2046, 2012.

32 Bethune GC, Mullen JB and Chang MC: HER2 testing of multifocal invasive breast carcinoma: how many blocks are enough? Am J Clin Pathol 140: 588-592, 2013.

33 Nakamura R, Song JP, Isogaki J, Kitayama Y and Sugimura H: Multiple (multicentric and multifocal) cancers in the ipsilateral breast with different histologies: profiles of chromosomal numerical abnormality. Jpn J Clin Oncol 33: 463-469, 2003.

34 Baranwal S and Alahari SK: Molecular mechanisms controlling E-cadherin expression in breast cancer. Biochem Biophys Res Commun 384: 6-11, 2009.

35 Klopp AH, Lacerda L, Gupta A, Debeb BG, Solley T, Li L, Spaeth E, Xu W, Zhang X, Lewis MT, Reuben JM, Krishnamurthy S, Ferrari M, Gaspar R, Buchholz TA, Cristofanilli M, Marini F, Andreeff $M$ and Woodward WA: Mesenchymal stem cells promote mammosphere formation and decrease E-cadherin in normal and malignant breast cells. PLoS One 5: e12180, 2010.

36 Weissenbacher T, Hirte E, Kuhn C, Janni W, Mayr D, Karsten U, Rack B, Friese K, Jeschke U, Heublein S, Dian D and Ditsch $\mathrm{N}$ : Multicentric and multifocal versus unifocal breast cancer: differences in the expression of E-cadherin suggest differences in tumor biology. BMC Cancer 13: 361, 2013. 